\title{
Diferentes tasas eVOlutivas entre GRUPOS DE ANGIOSPERMAS.
}

\section{EUDICOTILEDÓNEAS}

\author{
Susana Magallón Puebla
}

\author{
Departament of Geophysical Sciences, University of Chicago. 5734 S. Ellis Av.Chicago, IL, 60637, USA. \\ and The Field Museum. Roosevelt Road at Lake Shore Drive. Chicago, IL 60605, USA.
}

\begin{abstract}
Resumen. Se obtuvieron tres modelos de filogenias para dos clados de plantas, eudicotiledoneas y paleohierbas, utilizando modelos probabilísticos. Las filogenias se analizaron con diferentes de tasas de extinción y especiación. El Modelo de Filogenia I sugiere que la probabilidad de obtener una diferencia en la distribución de especies, entre los dos clados-hermanos, como la observada, o más extrema varía entre $15.2 \%$ con tasas de especiación elevadas, y $16.7 \%$ con tasas de extinción bajas. De acuerdo con el Modelo de Filogenia II, la probabilidad de obtener una distribución de especies como la observada o más extrema varía entre $15.7 \%$ con tasas de especiación elevadas, y $17.4 \%$ con tasas de extinción bajas. En el Modelo de Filogenia III la probabilidad de obtener una distribución de especies como la observada, o más extrema, varía de $2.5 \%$ con tasas de especiación elevadas, hasta 2.8 con tasas de extinción bajas. De acuerdo a los tres modelos las probabilidades de obtener una diferencia numérica al nivel de especie entre las eudicotiledóneas y su grupo-hermano son bajas. Sin embargo, los resultados sugieren que las eudicotiledónes están caracterizadas por tasas evolutivas (especiación - extinción) más elevadas que las de otros grupos de angiospermas.

Palabras clave: Angiospermas, eudicotiledóneas, especiación, extinción, tasas evolutivas.

Abstract. Applying analytical models to two clades, eudicotyledons and paleoherbs, three filogeny models were explored and analyzed with parameters that vary from low extinction rates to high speciation rates. Filogeny Model I suggest that the probability of obtaining a difference in species distribution, between the two sisterclades, as the observed, or higher, varies between $15.2 \%$ with high speciation rates and $16.7 \%$ with low extinction rates. In Filogeny Model II the probability of obtaining a species distribution similar to the observed one or higher, varies between $15.7 \%$ with high speciation rates to $17.4 \%$ with low extinction rates. In contrast, probability of obtaining a species distribution like the observed or higher in Filogeny Model III varies from $2.5 \%$ with high speciation rates to $2.8 \%$ with low extinction rates. Accordingly with the tree Models, the probability of obtaining a numerical difference at the species level between eudicotyledons and its sister group are low. However, results suggest the eudicotyledons are characterize by higher evolutionary rates (speciation-extinction) compared with other groups of angiosperms.
\end{abstract}

Key words: Angiosperms, eudicotyledons, evolutionary rates, extinction, speciation.

$\mathbf{L}$ as angiospermas dominan la vegetación actual de la mayoría de los ecosistemas terrestres, e incluyen un número de especies mayor que el resto de las plantas en conjunto. No sólo las angiospermas son más numerosas que algún otro grupo de plantas, vivas o extintas, sino que son más abundantes y diversas que sus parientes más cercanos, las Gnetales (Crane, 1985; Chase et al., 1993; Doyle et al., 1994; Rothwell y Serbert, 1994).

Las angiospermas son un grupo monofilético sumamente bien apoyado, tanto por caracteres morfológicos (e.g., Crane, 1985; Doyle y Donoghue, 1986; Loconte y Stevenson, 1990; Nixon et al., 1994; Rothwell y Serbert, 1994; Doyle et al., 1994) como por evidencias moleculares (e.g., Chase et al., 1993; Doyle et al., 1994). Las hipótesis de relaciones filogenéticas basadas tanto en datos morfológicos como moleculares indican la existencia de dos grandes clados dentro de 
Tabla 1: Distribución de especies en los principales grupos de angiospermas.

\begin{tabular}{|c|c|c|c|}
\hline $\begin{array}{l}\text { "Magnoliidae" } \\
\text { Taxa }\end{array}$ & $\begin{array}{r}\text { Número de } \\
\text { especies }\end{array}$ & $\begin{array}{l}\text { Eudicotiledóneas } \\
\text { Taxa }\end{array}$ & $\begin{array}{r}\text { Número de } \\
\text { especies }\end{array}$ \\
\hline Winteraceae & 90 & Theales & 5,135 \\
\hline Illiciaceae & 37 & Ericales & 2,650 \\
\hline Schisandracae & 47 & Fouquieriales & 11 \\
\hline Magnoliaceae & 220 & Styracales & 1,450 \\
\hline Degeneriaceae & 2 & Primulales & 2,870 \\
\hline Hamamelidales & 2 & Polygonales & 1,100 \\
\hline Eupomatiaceae & 2 & Celastrales & 875 \\
\hline Annonaceae & 2,300 & Malvales & 3,300 \\
\hline Myristicaceae & 300 & Urticales & 2,680 \\
\hline Canellaceae & 21 & Rhamnales & 900 \\
\hline Austrobaileyaceae & 1 & Euphorbiales & 7,730 \\
\hline Amborellaceae & 1 & Violales & 5,350 \\
\hline Trimeniaceae & 5 & Brassicales & 4,230 \\
\hline Monimiaceae & 335 & Batales & 2 \\
\hline Gomortegace & 1 & Santales & 1,995 \\
\hline Calycanthaceae & 7 & Balanophorales & 45 \\
\hline Lauraceae & 2,490 & Linales & 865 \\
\hline Hernandiaceae & 64 & Rhizophorales & 84 \\
\hline Total & 5,925 & Geraniales & 2,365 \\
\hline Paleohierbas & Número de & $\begin{array}{l}\text { Polygalales } \\
\text { Rutales }\end{array}$ & $\begin{array}{r}2,245 \\
24,300\end{array}$ \\
\hline Taxa & especies & Casuarinales & $\begin{array}{r}24,500 \\
70\end{array}$ \\
\hline Aristolochiaceae & 400 & $\begin{array}{l}\text { Balanopales } \\
\text { Bruniales }\end{array}$ & $\begin{array}{l}120 \\
115\end{array}$ \\
\hline Chloranthaceae & 70 & Juglandales & 100 \\
\hline Lactoridaceae & 1 & Betulales & 945 \\
\hline Saururaceae & 7 & Rosales & 2,050 \\
\hline Piperaceae & 2,000 & Saxifragales & 2,565 \\
\hline Cabomabaceae & 8 & Podostematales & 140 \\
\hline Nymphaeaceae & 62 & Cunoniales & 470 \\
\hline Monocotiledoneas & 58,178 & Hydrangeales & 485 \\
\hline Ceratophyllaceae & 6 & Cornales & 1,285 \\
\hline Total & 60,732 & Pittosporales & 195 \\
\hline Eudicotiledóneas & Número de & Dipsacales & 1,390 \\
\hline Taxa & especies & Asterales & 19,125 \\
\hline Berberidales & 3,490 & $\begin{array}{l}\text { Campanulales } \\
\text { Solanales }\end{array}$ & $\begin{array}{l}2,370 \\
7,735\end{array}$ \\
\hline Nelumbonales & 2 & Loasales & 280 \\
\hline Paeoniales & 34 & Myrtales & 8,120 \\
\hline Pioteales & 1,050 & Gentianales & 14,145 \\
\hline Hamamelidales & 136 & Scrophulariales & 17,790 \\
\hline Caryophyllales & 8,600 & Total & 167,249 \\
\hline
\end{tabular}

las angiospermas, que son las paleohierbas (incluyendo las monocotiledóneas) y las eudicotiledóneas (dicotiledóneas no relacionadas con Magnoliidae), y unos cuantos linajes parafiléticos relacionados con Magnoliidae (sensu Cronquist, 1981). El número de especies incluido en los grupos monofiléticos, así como en el grado basal de Magonliales, muestra que la diversi- dad de especies dentro de las angiospermas está distribuida inequitativamente.

Tradicionalmente, las plantas incluidas en Magnoliidae han sido consideradas similares a las angiospermas más ancestrales, y de acuerdo con ideas recientes, estas plantas conservan un conjunto amplio de características probablemente ancestrales, incluyendo una 
gran variedad de forma y arquitectura floral (Crane et al., 1995). Los grupos de plantas incluidos en el grado de las "magnoliales" en sentido amplio (el término "magnoliales" es usado informalmente, sin connotación ordinal), i.e., incluyendo Laurales, Illiciales, y Winterales, se enlistan en la tabla 1. El número de especies de cada taxón, tomado de Thorne (1992), indica que en total, hay 5,925 especies en el grado de las magnoliales, que corresponden al $2.5 \%$ de la abundancia actual de angiospermas. El registro fósil indica que las magnoliales se diversificaron tempranamente en la historia de las angiospermas, y que varias familias vivientes, tales como Lauraceae, Calycanthaceae, Magnoliaceae y tal vez Winteraceae y Eupomatiaceae, se habían diferenciado en el Turoniano (aproximadamente 90 millones de años antes del presente) (Crane et al, 1995, y referencias ahí citadas).

Las paleohierbas constituyen uno de los dos principales grupos monofiléticos de angiospermas. El clado de las paleohierbas incluye las monocotiledóneas, que constituyen un grupo monofilético caracterizado morfológicamente, entre otras cosas, por la presencia de inclusiones triangulares en los plástidos de los tubos cribosos, y por la producción de un solo cotiledón en el embrión (Dahlgren et al., 1985), y por varios linajes de dicotiledóneas herbáceas que tradicionalmente han sido relacionadas con Magnoliidae, entre los cuales se encuentran Chloranthaceae, Aristolochiaceae, Piperaceae y Nymphaeaceae. Los grupos incluidos en el clado de las paleohierbas se enlistan el la tabla 1. De acuerdo con el número de especies presentado por Thorne (1992), el clado de las paleohierbas incluye un total de 60,732 especies, que representan aproximadamente $26 \%$ del total de especies de angiospermas. No existe evidencia que documente la época del origen de las monocotiledóneas, sin embargo, este grupo se encuentra representado en la parte media del Cretácico, y aparentemente, se diversificó rápidamente durante el Cretácico Superior (Crane et al., 1995). Uno de los reportes de monocotiledóneas cretácicas consiste en frutos y semillas asignadas a Zingiberales, procedentes del Maastrictiano de Coahuila (Rodríguez de la Rosa y Cevallos Ferriz, 1994). Se ha documentado la existencia durante la parte más temprana del Cretácico Superior de algunas familias vivientes pertenecientes al clado de las paleohierbas, e.g., Chloranthaceae y Ceratophyllaceae (Crane et al., 1995). Los granos de polen de las paleohierbas tiene una sola abertura polar (granos monosulcados), muy similares a aquellos de magnoliales.

Quizás, algunos de los granos monosulcados más antiguos fueron producidos por plantas pertenecientes al clado de las paleohierbas. Las eudicotiledóneas constituyen un clado sumamente bien apoyado por evidencias tanto morfológicas como de secuencias moleculares (Crane, 1985; Donoghue y Doyle, 1989; Loconte y Stevenson, 1991; Chase et al., 1993; Doyle et al., 1994). En términos morfológicos, las eudicotiledóneas se caracterizan por producir granos de polen tricolpados, o derivados de éstos. Dentro de las eudicotiledóneas se encuentran algunas plantas que tradicionalmente han sido consideradas afines a Magnoliidae (sensu Cronquist, 1981), i.e., el orden Ranunculales (o Berberidales, según Thorne, 1992), así como las subclases Hamamelidae, Caryophyllidae, Dilleniidae, y las gigantescas Rosidae y Asteridae (todo en el sentido Cronquist, 1981). Los órdenes incluidos en el clado de las eudicotiledóneas, así como el número de especies que los constituyen, se enlistan en la tabla 1. Los nombres usados, así como los números de especies son en el sentido de Thorne (1992). Según lo anterior, hay 167,249 especies vivientes de eudicotiledóneas, que corresponden al $71.5 \%$ de la abundancia actual de angiospermas. La información respecto a la época de origen de las eudicotiledóneas está acoplada a la aparición de granos tricolpados en el registro fósil. De acuerdo con lo anterior, las eudicotiledóneas surgieron en alguna época cercana al Barremiano medio-tardío (aproximadamente 121 millones de años antes del presente), pues los granos tricolpados más antiguos proceden de estratos de ese tiempo (Crane, 1989; Doyle y Donghue, 1993).

Debido a su abundancia en términos de número de especies y número de individuos, y debido a que se les encuentra prácticamente en todos lados, las angiospermas han sido consideradas como un grupo de organismos extremadamente exitoso. Sin embargo, dada la distribución de especies dentro de las angiospermas, es claro que las eudicotiledóneas son responsables, en gran medida, de esa abundancia y diversidad. Si las eudicotiledóneas no hubieran surgido, ¿cuán abundantes y diversas serían las angiospermas? Es decir, ¿son las angiospermas abundantes y diversas sólo porque las eudicotiledóneas son abundantes y diversas? Cuando hablamos de la riqueza de las angiospermas, ¿nos referimos en realidad a la riqueza de eudicotiledóneas? La respuesta inmediata a estas preguntas parece ser negativa, en parte porque dos de las familias más grandes de angiospermas (i.e., Poaceae y Orchidaceae) no son eudicotiledóneas. Otro tipo de argumento se refiere a la época en que ocurrió la radiación evolutiva de las angiospermas. Si hubiese un acoplamiento entre el origen de las eudicotiledóneas y la radiación evolutiva de las angiospermas, habría argumentos importantes para igualar el éxito evolutivo de las angiospermas con el éxito evolutivo de las eudicotiledóneas. Sin embargo, no existe tal acoplamiento. Las eudicotiledóneas surgieron 
en el Barremiano, antes de que ocurriera la radiación evolutiva de las angiospermas entre el Albiano y el Aptiano ( $c f$. Grace, 1989). La radiación evolutiva de las angiospermas incluyó las magnoliales, las palohierbas y las eudicotiledóneas. Sin embargo, no existe ninguna aproximación respecto a la contribución de cada uno de estos grupos en la radiación evolutiva de las angiospermas. No es claro si la radiación evolutiva corresponde a todas las angiospermas, o sólo a algunos de los grupos monofiléticos dentro de las angiospermas.

Algunos autores (e.g., Doyle y Donoghue, 1993) han planteado la pregunta de si las características que promueven la abundancia de especies son comunes a todas las angiospermas (i.e., sinapomorfias al nivel de las angiospermas), o bien, caracterizan solamente a un subgrupo de plantas con flor (i.e., sinapomorfias de un clado dentro de las angiospermas).

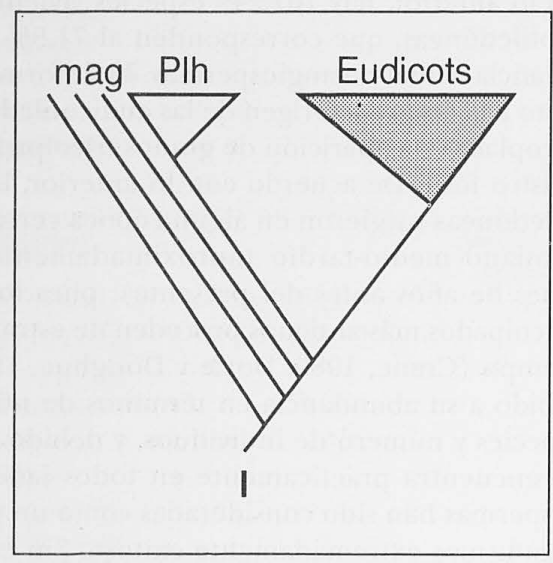

Figura 1. Filogenia obtenida de Doyle y Donoghue, (1989) y Laconte y Stevenson (1991).

Algunos autores que han abordato el tema de la abundancia de las angiospermas han enfocado sus investigaciones a la posible distribución diferencial dentro de las angiospermas de características que promueven grandes números de especies (i.e., Doyle y Donoghue, 1993), o bien, en la abundancia y tasa de especiación diferencial entre las angiospermas y su grupo-hermano (i.e., Sanderson y Donoghue, 1994). El presente trabajo explora la posible distribución diferencial de tasas de especiación y extinción dentro de las angisopermas como la causa de la diferencia numérica observada entre los clados de angiospermas. La abundancia de las eudicotiledóneas es el principal punto de interés. ¿Es posible explicar la abundancia numérica de las eudicotiledóneas considerando una sola tasa de especiación y una sola tasa de extinción para todas las angiospermas? En este trabajo pretendo explorar, y dar una aproximación a la siguiente pregunta: si consideramos una sola tasa de especiación y una sola tasa de extinción, ¿cuál es la probabilidad de obtener una diferencia en número de especies tan grande o más grande que la observada entre el clado de las eudicotiledóneas y su grupo hermano? Sanderson y Donoghue (1994) hicieron una pregunta similar respecto a la diferencia numérica entre las angiospermas y su grupo hermano, i.e., las gnetales. Estos autores utilizaron un método de máxima posibilidad (maximum likelihood), y utilizaron un modelo en el que sólo se considera especiación (i.e., "pure birth", Yule, 1924). En el presente trabajo, se usa un enfoque analítico, y se consideran tanto tasas de especiación como tasas de extinción.

\section{La filogenia de las angiospermas: ¿cuál es el grupo-hermano de las eudicotiledóneas?}

Mientras que la monofilia de las angiospermas es clara, y según la mayoría de los análisis filogenéticos un clado que incluye a todas las gnetales es el grupo hermano de las angiospermas, las relaciones filogenéticas entre los principales grupos de angiospermas no han sido resueltas. Uno de los principales cuestionamientos se refiere a la posición de la raíz del árbol de las angiospermas. Donoghue y Doyle (1989), haciendo uso de evidencia de tipo morfológico, presentaron un árbol más parsimonioso que tiene su raíz cerca de los grupos de magnoliales. Sin embargo, estos autores encontraron que hipótesis de relaciones filogenéticas que son uno o dos pasos más largas, están enraizadas cerca de las paleohierbas (Donoghue y Doyle, 1989; Doyle y Donoghue, 1993). Loconte y Stevenson

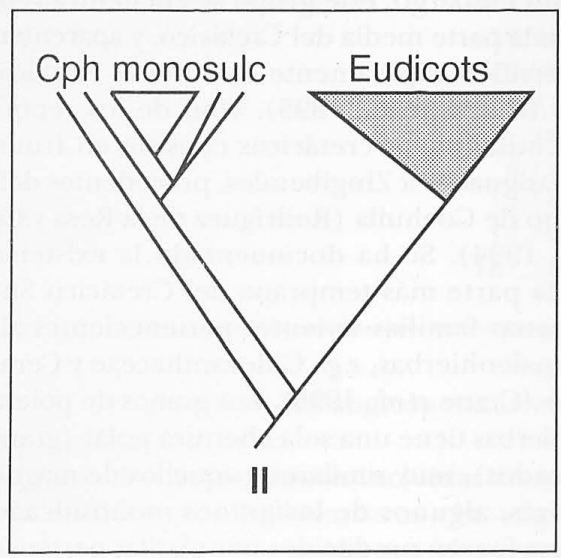

Figura 2. Filogenia obtenida de Chase et al. (1993). 
(1991), utilizando datos morfológicos y químicos, obtuvieron una hipótesis de relación filogenética similar a la más parsimoniosa de Donoghue y Doyle (1989), en el sentido que encontraron una serie de linajes parafiléticos relacionados con magnoliales cerca de la base, y dos grandes clados en relación de grupos-hermanos: las paleohierbas y las eudicotiledóneas (figura 1).

Otro tipo de estructura en las relaciones filogenéticas de las angiospermas fue obtenida por Chase $e t$ al.

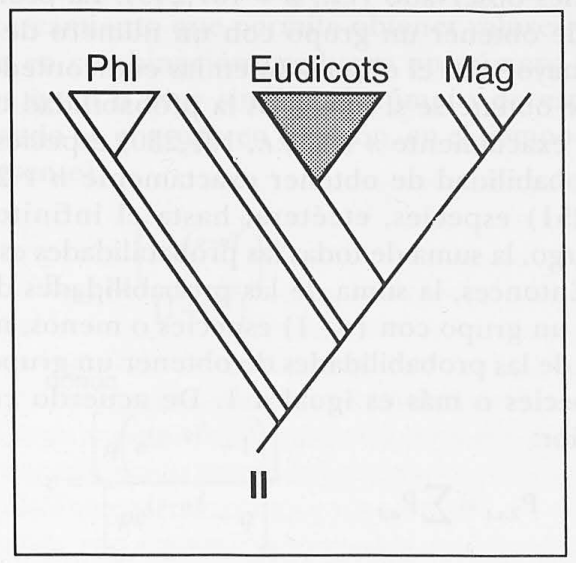

Figura 3. Filogenia tomada de Doyle et al. (1994).

(1993), quienes, con base en evidencia de secuenica molecular de $r b c L$, encontraron que la rama más basal de las angiospermas separa al género Ceratophyllum, que es grupo-hermano del resto de las angiospermas. El principal agrupamiento de las angiospermas incluye dos clados. Uno de estos incluye las plantas que producen granos de polen monosulcados o derivados, abarcando las magnoliales, paleohierbas y monocotiledóneas. El otro clado incluye a las plantas productoras de granos tricolpados, o derivados, de éstos, es decir las eudicotiledóneas (figura 2).

Una hipótesis de relación filogenética reciente, producida usando datos morfológicos y de secuencia ARN ribosomal (Doyle et al., 1994) presenta un esquema en el que en la base del árbol de las angiospermas se encuentran varios linajes parafiléticos de paleohierbas y las monocotiledóneas. Las eudicotiledóneas son el grupo-hermano de un clado que abarca las magnoliales (figura 3 ).

Cada una de estas hipótesis de relación filogenética indica un grupo-hermano diferente para las eudicotiledóneas. De acuerdo con el árbol más parsimonioso de Donoghue y Doyle (1989) y con Loconte y Stevenson (1991), las paleohierbas son el grupo-hermano de las eudicotiledóneas (figura 1). Según Chase et al.
(1993), el resto de las angiospermas con excepción de Ceratophyllum, forman un clado que es el grupohermano de las eudicotiledóneas (figura 2). Y de acuerdo con Doyle et al. (1994), un grupo monofilético de Magnoliales es el grupo hermano de las eudicotiledóneas (figura 3). De acuerdo con lo anterior, se establecerán tres comparaciones distintas, una para cada supuesto grupo-hermano de las eudicotiledóneas. El esquema de Donoghue y Doyle (1989), y el de Loconte y Stevenson (1991) constituye el Modelo I, en el que las paleohierbas son el grupo-hermano de las eudicotiledóneas. El esquema de Chase et al. (1993) constituye el Modelo II, en el que el resto de las angiospermas, con excepción de Ceratophyllum, son el grupo-hermano de las eudicotiledóneas, y el esquema de Doyle et al. (1994) es el Modelo III, en el que un clado de magnoliales es el grupo-hermano de las eudicotiledóneas.

\section{Métodos}

¿Cuál es la probabilidad de obtener una diferencia en el número de especies tan o más grande que la que se observa entre las eudicotiledóneas y su grupo-hermano?

La probabilidad de que exista una diferencia en el número de especies de la magnitud observada entre las eudicotiledóneas y su grupo-hermano, o mayor que ésta, será explorada a través de un método analítico. D. M. Raup proporcionó una serie de ecuaciones que permiten caracterizar a los clados por sus propiedades fundamentales de ramificación, i.e., por su tasa de especiación ( $p$ ) y su tasa de extinción $(q)$ (Raup, 1985). Estas tasas se refieren al número de eventos (especiación o extinción) por unidad de tiempo, que convencionalmente se ha establecido como un millón de años. En estos modelos, se considera que para cada clado, existen valores promedio alrededor de los cuales la magnitud de $p$ y de $q$ varían al azar, y que $p$ y $q$ son aproximadamente constantes a lo largo del tiempo.

La pregunta que se pretende resolver es si los dos taxa-hermanos están caracterizados por tasas de especiación y tasas de extinción iguales o diferentes. La hipótesis nula mantiene que las tasas de especiación y las tasas de exitinción de ambos grupos-hermanos son similares. Una manera de resolver esta pregunta consiste en obtener la probabilidad combinada de que el taxón que tiene un menor número de especies tenga exactamente el número de especies observado, o menor, y que su taxón-hermano tenga exactamente el número de especies observado, o mayor. Por ejemplo, en el Modelo I, de acuerdo con el cual las paleohierbas son el grupo-hermano de las eudicotiledóneas, calcularemos la probabilidad de que las paleohierbas 
tengan exactamente 60,732 especies (tabla 1), o menos, y que las eudicotiledóneas tengan exactamente 167,249 especies (tabla 1), o más. Al multiplicar las dos probabilidades anteriores, se obtiene la probabilidad de que exista una distribución en el número de especies como la observada, o más extrema.

Raup(1985) presenta una ecuación para obtener la probabilidad de que un taxón tenga exactamente un número de especies $(n)$, en un tiempo $(t)$ posterior al origen del grupo (evento de ramificación), dada una tasa de especiación $(p)$ y una tasa de extinción (q) $(\operatorname{con} p \neq q)$ que caracterizan a ese taxón (ecuación A 17; Raup, 1985). La ecuación de Raup incluye una expresión que calcula la probabilidad de que el taxón en cuestión exista en el tiempo $t$, es decir, que ese taxón no esté extinto en el tiempo $t$. Sin embargo, sabemos con certeza que los taxa que estamos analizando (i. e., las eudicotiledoneas y sus posibles grupos hermanos) existen en el tiempo $t$ (i.e., el presente). Foote (1991) desarrolló una ecuación, basada en la expresión de Raup, que calcula la probabilidad de que un taxón tenga exactamente un número $n$ de especies en el tiempo $t$, teniendo la certeza de que el taxón existe en el tiempo $t$ :

$$
\begin{aligned}
& \mathrm{P}_{n, t}=(1-b) b^{n-1} \\
& \text { donde } \\
& b=\left(\frac{p}{q}\right)\left[\frac{q\left(e^{(p-q)^{t}}-1\right)}{p e^{(p-q)^{t}}-q}\right]
\end{aligned}
$$

$p=$ tasa de especiación (en número de eventos por linaje por millón de años).

$q=$ tasa de extinción (en número de eventos por linaje por millón de años).

$t=$ tiempo transcurrido desde el origen del grupo (en millones de años).

Mediante el uso de esta ecuación, podemos obtener la probabilidad exacta de que el grupo-hermano de las eudicotiledóneas, i.e., las paleohierbas en el Modelo I, tengan exactamente el número de especies observado (i.e., $n=60,732$ spp.). Sin embargo, no sólo nos interesa conocer esa probabilidad, sino también queremos saber cuál es la probabilidad de que las paleohierbas tengan un número de especies menor que el observado. Por lo tanto, podemos calcular cuál es la probabilidad exacta de que el grupo en cuestión tenga exactamente 1 especie en el tiempo $t$, de que tenga exactamente 2 especies en el tiempo $t$, de que tenga exactamente 3 especies en el tiempo $t, \ldots$, y que tenga exactamente ( $n$-1) especies (i.e., 60,731) en el tiempo $t$. La suma de estas probabilidades re- presenta la probabilidad de tener un grupo del tamaño de las paleohierbas, o más pequeño, en el tiempo $t$. La siguiente ecuación expresa la probabilidad de obtener un grupo igual o menor que $n$, en el tiempo $t$ :

$$
\mathrm{P}_{\leq n, t}=\sum \mathrm{P}_{n, t}
$$

De manera similar, mediante el uso de la ecuación (1), podemos calcular la probabilidad de que las eudicotiledóneas tengan exactamente el número de especies observado (i.e., $n=167,249$ ). La probabilidad de obtener un grupo con un número de especies mayor que el observado en las eudicotiledóneas puede obtenerse si sumamos la probabilidad de obtener exactamente $n+1$ (i.e., 167,250) especies, más la probabilidad de obtener exactamente $n+2$ (i.e., 167,251) especies, etcétera, hasta el infinito. Sin embargo, la suma de todas las probabilidades es igual a 1. Entonces, la suma de las probabilidades de obtener un grupo con $(n-1)$ especies o menos, más la suma de las probabilidades de obtener un grupo con $n$ especies o más es igual a 1 . De acuerdo con lo anterior:

$$
\begin{aligned}
\mathrm{P}_{\geq n . t} & =\sum \mathrm{P}_{n, t} \\
& =1-\mathrm{P}_{\leq(n-1) t}
\end{aligned}
$$

La probabilidad de que el taxón-hermano más pequeño tenga el número observado de especies o menos, y que el taxón-hermano más grande tenga el número observado de especies o más (i.e., que la distribución del número total de especies sea igual o más extrema que la observada), es el producto de las ecuaciones (2) y (3). Este producto expresa la probabilidad de que un grupo en particular tenga un número $x$ de especies y de que el otro grupo en particular tenga un número y de especies. Sin embargo, nuestro interés se refiere a la distribución del número total de especies en dos grupos, y no a que uno de estos grupos en particular tenga uno u otro número de especies (distribución de una cola). Por lo tanto, el valor que se obtenga debe duplicarse, para obtener la probabilidad (distribución de una cola) de tener cualquiera de los grupos-hermanos con un número de especies $n$ o menor, y el otro grupo, con un número de especies $n$ o mayor (Foote, 1991).

$$
\mathrm{P}_{\text {distribución observaldat }}=\left(\mathrm{P}_{\leq n, t} \mathrm{P}_{\geq n, t}\right) 2
$$

Todas las ecuaciones anteriores requieren que conozcamos c tiempo transcurrido en millones de años desde el origen de los grupos en cuestión, así 
como la tasa de especiación ( $p$ ) y la tasa de extinción (q). El registro fósil puede proporcionar una aproximación aceptable respecto al tiempo de origen de los clados dentro de las angiospermas. Sin embargo, varios factores combinados, entre los cuales se encuentran la preservación fragmentaria de las plantas, así como los problemas en la identificación de restos fósiles fragmentarios, impiden que se tenga al menos una idea remota acerca de las tasas de especiación y extinción de las angiospermas, o de los subgrupos incluidos en éstas. Sin embargo, Foote (1991) desarrolló un acercamiento que permite obtener valores de $p$ y $q$, que en combinación producen un número de especies igual o muy similar al número de especies observado en el grupo en cuestión, en el tiempo $t$ (i.e., el presente):

$$
\mathrm{E}_{(n, t)}=\frac{\left[m e^{(p-q)^{t}}\right]}{\left(1-c^{m}\right)}
$$

donde

$$
c=\frac{\left[q\left(e^{(p-q)^{t}}-1\right)\right]}{\left[p e^{(p-q)^{t}}-q\right]}
$$

$\mathrm{E}_{(n, t)}=$ número de especies esperado en el tiempo $t$. $m=$ número de especies en el tiempo cero.

En este esquema, se está haciendo la suposición de que el número de especies observadas en el presente es igual al número esperado para ese clado. El tiempo cero corresponde al instante inmediatamente posterior al evento de ramificación que originó a los grupos hermanos en cuestión. En el tiempo cero, el número de especies $(m)$ es igual a 2; una correspondiente a cada una de las ramas producidas en el evento de ramificación. El tiempo transcurrido desde el evento de ramificación puede ser obtenido a partir del registro fósil, suponiendo que la primera aparición en el registro fósil del grupo más antiguo es cercana al evento de ramificación que dio origen a ambos linajes. Bajo tales suposiciones, es posible obtener un inmenso número de combinaciones para los valores de $p$ y $q$, que resulten en un número esperado de especies en el tiempo $t$, cercano al número observado (e.g., $\pm 1 \%$ ). Si fijamos una de las dos variables en algún valor arbitrario, (e.g., $\mathrm{p}=0.3$ ), podemos obtener el valor correspondiente de $q$, de tal manera que el valor de $\mathrm{E}_{(n, t)}$ corresponda a $\pm 1 \%$ del número de especies correspondiente a la suma del número de especies de los dos grupos-hermanos. Por ejemplo, en el Modelo de ramificación I, y de acuerdo con los datos de la tabla 1, la suma del nú- mero de especies de los dos grupos-hermanos involucrados (eudicotiledóneas, con 167,249 especies, y paleohierbas, con 60,732 especies) es igual a 227,981 especies. El intervalo entre $\pm 1 \%$ del número de especies observado se encuentra entre 225,701 y 230,261 especies. Con base en la primera aparición en el registro fósil de granos tricolpados (i.e., Barremiano medio-tardío), consideramos que la ramificaicón de los grupos-hermanos ocurrió 121 millones de años antes del presente $(t=121)$. Si fijamos el valor de $p$ (o de $q$ ), podemos obtener el valor de $q$ (o de $p$ ) que en la ecuación (5) brinde un valor de $\mathrm{E}_{(n, t)}$ que se encuentre entre 225,701 y 230,261.

Cada uno de los modelos de relación filogenética para las eudicotiledóneas será examinado independientemente. Para cada modelo, la suma del número de especies de los dos grupos-hermanos será tomado como el número esperado de especies. Se obtendrán valores de $p$ y q que expliquen adecuadamente la abundancia numérica de ambos grupos. Los valores obtenidos de $p$ y $q$ son aproximaciones a los valores necesarios para explicar la abundancia numérica combinada de los dos grupos-hermanos involucrados en cada modelo, sin considerar la diferencia en el tamaño de los clados.

Existe la noción de que las angiospermas tienen tasas de especiación elevadas. De acuerdo con lo anterior, el valor de $p$ fue fijado en 0.6 y 0.8 , y se obtuvieron los valores correspondientes de $q$. Sin embargo, el hecho de que el registro fósil de angiospermas no parezca mostrar familias extintas parece indicar que las angiospermas tienen tasas de extinción muy bajas. Por tanto, en adición a los valores altos de $p$, el valor de $q$ fue fijado en 0.2 y en 0.4 , obteniéndose los valores correspondientes de $p$. La tabla 2 muestra, para cada modelo de relación filogenética, las combinaciones de valores $p$ y $q$ que producen un número de especies esperado correspondiente a $\pm 1 \%$ del número observado del total de los dos gruposhermanos.

En los modelos de filogenia I y III (i.e., Doyle y Donoghue, 1989; Loconte y Stevenson, 1991; Doyle et al., 1994), el tiempo en que ocurrió la ramificación que originó los grupos hermanos es establecido en 121 millones de años antes del presente (ver arriba). En estos modelos, es posible suponer que los granos de polen monosulcados de estratos más antiguos que 121 millones de años fueron producidos por magnoliales, en los modelos de filogenia de Donoghue y Doyle (1989), o por paleohierbas, en el modelo de filogenia de Doyle et al. (1994). Bajo el modelo de Chase et al. (1993), hay una diferencia en el tiempo de origen de las eudicotiledóneas y su grupo-hermano. Mientras que los granos tricolpados aparecen en el Barremiano, los 
Tabla 2: Obtención de tasas de especiación (p) y tasas de extinción (q).

\begin{tabular}{|c|c|c|c|c|c|c|}
\hline $\begin{array}{l}\text { Modelo de } \\
\text { filogenia }\end{array}$ & $t$ & $\begin{array}{r}N \\
\pm 1 \% \\
\end{array}$ & & & & \\
\hline $\begin{array}{l}\quad \text { I } \\
\text { Eudicots. vs } \\
\text { Paleohierbas }\end{array}$ & 121 & $\begin{array}{r}227,981 \\
225,701-230,261\end{array}$ & $\begin{array}{l}p=0.291 \\
q=0.2 \\
E=229,459.4\end{array}$ & $\begin{array}{r}0.487 \\
0.4 \\
229,326.1\end{array}$ & $\begin{array}{r}0.6 \\
0.5148 \\
227,464.6\end{array}$ & $\begin{array}{r}0.8 \\
0.7173 \\
226,188.9\end{array}$ \\
\hline $\begin{array}{l}\quad \text { II } \\
\text { Eudicots. vs. } \\
\text { monosulcs. }\end{array}$ & 127 & $\begin{array}{r}233,900 \\
231,561-236,239\end{array}$ & $\begin{array}{l}p=0.2866 \\
q=0.2 \\
E=232,994.2\end{array}$ & $\begin{array}{r}0.4827 \\
0.4 \\
232,492\end{array}$ & $\begin{array}{r}0.6 \\
0.519 \\
233,127.9\end{array}$ & $\begin{array}{r}0.8 \\
0.7213 \\
234,279.1\end{array}$ \\
\hline $\begin{array}{l}\quad \text { III } \\
\text { Eudicots. vs. } \\
\text { magnoliales }\end{array}$ & 121 & $\begin{array}{r}173,174 \\
171,442-174,906\end{array}$ & $\begin{array}{l}p=0.2885 \\
q=0.2 \\
E=172,246.8\end{array}$ & $\begin{array}{r}0.4845 \\
0.4 \\
173,178.7\end{array}$ & $\begin{array}{r}0.6 \\
0.5173 \\
172,781.2\end{array}$ & $\begin{array}{r}0.8 \\
0.7198 \\
172,071\end{array}$ \\
\hline
\end{tabular}

granos monosulcados aparecen cọn anterioridad, pues están presentes en estratos del Hauteriviano medio (Crane, 1989). Esto implica que la ramificación que dio origen a ambos grupos ocurrió en un tiempo cercano a la aparición de granos monosulcados en el registro fósil. Lo anterior significa que el período inicial en la historia de las eudicotiledóneas no quedó marcado en el registro fósil, y que su origen debe extenderse hasta una época previa al primer registro de granos tricolpados, mediante un linaje fantasma. Los linajes fantasma son "segmentos de historia evolutiva que pueden ser obtenidos no sólo del registro fósil, sino a través de hipótesis de relaciones filogenéticas que han sido calibrados con datos del registro fósil" (Norell, 1993; Wagner, 1995). De acuerdo con lo anterior, en el modelo de filogenia II, el tiempo transcurrido desde la ramificación fue fijado en 127 millones de años antes del presente (tabla 2).

Una vez que, de acuerdo con cada modelo de filogénia han sido obtenidos los parámetros de especiación y extensión para las eudicotiledóneas y su grupo-hermano, es posible insertar tales parámetros en la ecuación (4). Esto permite obtener la probabilidad exacta de obtener una diferencia en la distribución en el número de especies como la observada, o mayor, entre los grupos-hermanos.

\section{Resultados}

Probabilidad de la diferencia en el número de especies en los taxa-hermanos.

La tabla 3 muestra la probabilidad de obtener, bajo las mismas tasas de especiación y de extinción, i) un clado tan o más grande que las eudicotiledóneas, ii) un clado tan o más pequeño que el grupo-hermano de las eudicotiledóneas, y iii) una diferencia en la distribución de especies tan o más extrema que la observada. Las probabilidades fueron obtenidas para cada modelo de filogenia y, en cada caso, se analizaron los resultados de acuerdo con parámetros que varían desde bajas tasas de extinción (i.e., $q=0.2$ ), hasta altas tasas de especiación (i.e., $p=0.8$ ).

Bajo el Modelo de filogenia I (Doyle y Donoghue, 1989; Loconte y Stevenson, 1991), los resultados indican que la probabilidad de obtener una diferencia en la distribución de especies entre los dos cladoshermanos como la observada, o más extrema, varía entre $15.2 \%$, con tasas de especiación elevadas, y $16.7 \%$, con tasas de extinción bajas. De acuerdo con el Modelo de filogenia II (Chase et al., 1993), la probabilidad de obtener una distribución de especies como la observada o más extrema, varía entre $15.7 \%$, con tasas de especiación elevadas, y $17.4 \%$, con tasas de extinción bajas. En el Modelo de filogenia III (Doyle et al., 1994), la probabilidad de obtener una distribución de especies como la observada, o más extrema, varía de $2.5 \%$, con tasas de especiación elevadas, hasta 2.8 , con tasas de extinción bajas.

\section{Discusión}

Los valores obtenidos para las tasas de especiación y de extinción, que aparecen en la tabla 2, muestran que la diferencia entre el valor de $p$ y el valor de $q$ es pequeña (i.e., menor que 0.1), y que la magnitud de esta diferencia es aproximadamente constante tanto en los difere tes modelos de filogenia, como al considerar valores elevados de especiación y valores ba- 
jos de extinción. Los resultados obtenidos son consistentes con la cercanía en magnitud esperada para los valores de tasa de especiación $(p)$ y de extinción $(q)$, de acuerdo con la fluctuación de la diversidad alrededor de un valor de equilibrio (Valentine, 1990).

Modelo de filogenia I. En los modelos de relación filogenética para las angiospermas en los que hay una relación de grupo-hermano entre las eudicotiledóneas y las paleohierbas (Donoghue y Doyle, 1989; Loconte y Stevenson, 1991), la probabilidad de obtener una diferencia en la distribución de especies entre ambos grupos, dada una misma tasa de especiación y tasa de extinción, es baja (15.2 a $16.7 \%$ ). Lo anterior sugiere que las eudicotiledóneas y las paleohierbas están caracterizadas por parámetros evolutivos diferentes. Aunque en esta comparación no están incluidos los grupos relacionados con magnoliales, el bajo número de especies vivientes en estos grupos, así como los resultados de la comparación entre eudicotiledóneas y paleohierbas, que incluyen un número de especies mucho mayor que las magnoliales, sugiere que las características que confieren altas tasas evolutivas son propias de un clado dentro de las angiospermas (i.e., eudicotiledóneas), pero no de las angiospermas como un todo.

Modelo de filogenia II. La comparación entre las eudicotiledóneas y su grupo hermano, que según el modelo de filogenia de Chase et al. (1993), incluye al resto de las angiospermas con excepción de Cerato- phyllum, resulta la más equitativa en términos del número de especies en cada uno de los clados involucrados. Sin embargo, la probabilidad de obtener la distribución de especies observadas en ambos grupos, dada una misma tasa de especiación y una misma tasa de extinción, es baja (15.7 a $17.4 \%)$. Esta comparación sugiere que las eudicotiledóneas poseen una tasa evolutiva más elevada que la del resto de las angiospermas. Las tasas de especiación y de extinción que brindan los números elevados de especies, caracterizan un solo clado dentro las angiospermas (i.e., las eudicotiledóneas), y no a todas las angiospermas. Si este modelo de filogenia es correcto, parece atinado hablar, no de la abundancia y diversidad de las angiospermas, sino de las eudicotiledóneas.

Modelo de filogenia III. Bajo la hipótesis de relación filogenética que indica que las magnoliales en sentido amplio constituyen el grupo hermano de las eudicotiledóneas, la probabilidad de obtener una diferencia en la distribución de especies como la observada es sumamente baja $(2.5$ a $2.8 \%)$. El resultado anterior sugiere que la tasa evolutiva que caracteriza a las eudicotiledóneas es mayor que la de su grupohermano. La topología del árbol filogenético de las angiospermas (Doyle et al., 1994) muestra que en la base se encuentran varios linajes de paleohierbas y las monocotiledóneas (grado basal de paleohierbas). Cada uno de los linajes de paleohierbas incluyen un número pequeño de especies, sin embargo, las mo-

Tabla 3: Probabilidad de obtener grupos-hermanos con el número de especies observado, y de obtener una diferencia en la distribución de especies como la observada, o más extrema.

\section{Modelo de \\ ramificación}

\begin{tabular}{|c|c|c|c|c|}
\hline \begin{tabular}{l}
\multicolumn{1}{c}{} \\
Eudicots vs. \\
paleohierbas
\end{tabular} & $\begin{array}{l}p=0.291 \\
q=0.2 \\
P>=\text { Euds }=0.3640 \\
P<=P l h=0.2288 \\
\mathbf{P P}=\mathbf{0 . 1 6 6 6}\end{array}$ & $\begin{array}{l}p=0.487 \\
q=0.4 \\
P>=\text { Euds }=0.3593 \\
P<=P l h=0.2169 \\
\mathbf{P P}=\mathbf{0 . 1 5 5 9}\end{array}$ & $\begin{array}{l}p=0.6 \\
q=0.5148 \\
P>=\text { Euds }=0.3580 \\
P<=P l h=0.2141 \\
\mathbf{P P}=\mathbf{0 . 1 5 3 3}\end{array}$ & $\begin{array}{l}p=0.8 \\
q=0.7173 \\
P>=\text { Euds }=0.3572 \\
P<=P I h=0.2127 \\
\mathbf{P P}=\mathbf{0 . 1 5 2 0}\end{array}$ \\
\hline $\begin{array}{l}\quad \text { II } \\
\text { Eudictos vs. } \\
\text { monosulc. }\end{array}$ & $\begin{array}{l}p=0.2866 \\
q=0.2 \\
P>=\text { Euds }=0.3628 \\
P<=\text { mns }=0.2401 \\
\mathbf{P P}=\mathbf{0 . 1 7 4 2}\end{array}$ & $\begin{array}{l}p=0.4827 \\
q=0.4 \\
P>=\text { Euds }=0.3588 \\
P<=\text { mns }=0.2323 \\
\mathbf{P P}=\mathbf{0 . 1 6 5 3}\end{array}$ & $\begin{array}{l}p=0.6 \\
q=0.529 \\
P>=\text { Euds }=0.3568 \\
P<=\text { mns }=0.2264 \\
\mathbf{P P}=\mathbf{0 . 1 6 1 6}\end{array}$ & $\begin{array}{l}p=0.8 \\
q=0.7213 \\
P>=\text { Euds }=0.3543 \\
P<=\text { mns }=0.2217 \\
\mathbf{P P}=\mathbf{0 . 1 5 7 1}\end{array}$ \\
\hline $\begin{array}{l}\quad \text { III } \\
\text { Eudictos vs. } \\
\text { Magnoliales }\end{array}$ & $\begin{array}{l}p=0.2855 \\
q=0.2 \\
P>=\text { Euds }=0.3641 \\
P<=\text { mags }=0.0392 \\
\mathbf{P P}=\mathbf{0 . 0 2 8 5}\end{array}$ & $\begin{array}{l}p=0.4845 \\
q=0.4 \\
P>=\text { Euds }=0.3673 \\
P<=\text { mags }=0.0362 \\
\mathbf{P P}=\mathbf{0 . 0 2 6 6}\end{array}$ & $\begin{array}{l}p=0.6 \\
q=0.5173 \\
P>=\text { Euds }=0.3676 \\
P<=\text { mags }=0.0354 \\
\mathbf{P P}=\mathbf{0 . 0 2 6 0}\end{array}$ & $\begin{array}{l}p=0.8 \\
q=0.7198 \\
P>=\text { Euds }=0.3678 \\
P<=\text { mags }=0.0347 \\
\mathbf{P P}=\mathbf{0 . 0 2 5 5}\end{array}$ \\
\hline
\end{tabular}


nocotilidóneas tienen una abundancia elevada de especies. Según este modelo de filogenia, es más parsimonioso suponer que las tasas evolutivas altas, que producen linajes con números de especies elevados, surgieron independientemente en las monocotiledóneas y en las eudicotiledóneas. Tales tasas evolutivas altas caracterizan sólo algunos clados dentro de las angiospermas (e.i., las monocotiledóneas y las eudicotiledóneas), pero no a las angiospermas como un todo.

Sin embargo, inaginemos que existe una topología en la que un clado de paleohierbas (opuesto a un grado), existiese en la base de las angiospermas (figura 4A). Bajo tal filogenia, es igualmente parsimonioso suponer que las tasas evolutivas altas (que producen grupos del tamaño del clado de las paleohierbas, y del tamaño de las eudicotiledóneas), caracterizan a todas las angiospermas, pero fueron perdidas en el linaje de las magnoliales, (transformación acelerada) (figura 4b) o bien que las tasas evolutivas elevadas fueron adquiridas independientemente en el linaje de la paleohierbas, y en el linaje de las eudicotiledóneas (transformación retardada) (figura 4C). De acuerdo con la primera suposición, es posible interpretar las tasas evolutivas elevadas como propias del clado de las angiospermas.

Los resultados obtenidos dependen del cumplimiento de tres suposiciones. La primera ha sido ya mencionada, y se refiere a que los valores de las tasas de evolución y de extinción fueron obtenidos suponiendo que el proceso evolutivo real brindó el número esperado (i.e., observado) de especies. La segunda suposición se refiere a que las tasas de especiación y de extinción se mantienen constantes a lo largo del tiempo. La tercera suposición considera que no han ocurrido eventos de extinción masiva (no atribuible a la tasa de extinción propia del grupo) en la historia de las angiospermas. Sin embargo debido a la naturaleza estocástica y taxonómicamente no específica que caracteriza a los eventos de extinción masiva, es probable que un posible evento de extinción masiva (no detectado por el registro fósil) afectase a los clados de angiospermas por igual. En este caso, las tasas evolutivas calculadas para los grupos-hermanos serían inferiores a las reales.

\section{Conclusiones}

De acuerdo a los tres modelos de filogenia generalizados para las angiospermas, las probabilidades de obtener una diferencia numérica al nivel de especie entre las eudicotiledóneas y su grupo-hermano son bajas. Los resultados sugieren que las eudicotiledóneas están caracterizadas por tasas evolutivas (especiación- extinción) más elevadas que las de otros grupos de angiospermas.

La técnica analítica empleada permite identificar linajes en los cuales hay tasas evolutivas elevadas. Sin embargo, no brinda ninguna explicación causal para tales tasas evolutivas. Si bien cada clado está caracterizado por la presencia de caracteres derivados compartidos (i.e., sinapormofias), la interpretación de éstos como innovaciones clave requiere de una evaluación rigurosa de las implicaciones ecológicas y evolutivas de tales sinapomorfias. Por otra parte, no existe una correlación implícita entre el surgimiento innovaciones clave, y la presencia de tasas evolutivas elevadas. La supuesta correlación entre las innovaciones clave y las tasas evolutivas elevadas debe ponerse a prueba, y sin embargo, este es un aspecto que ha sido explorado apenas superficialemente.

\section{Agradecimientos}

El presente estudio es el resultado de un proyecto realizado para el curso de "Paleobiología Analítica", impartido por el Dr. Mike Foote, en la Universidad de Chicago. Quisiera agradecer extensamente al Dr. Peter Crane, por la inspiración inicial, y al Dr. Mike Foote, por dar el ímpetu y la asesoría necesaria para que este trabajo se llevara a cabo. La autora recibe apoyo económico de DGAPA, UNAM, para realizar estudios de posgrado.

\section{Literatura citada}

Chase M.W., Soltis D.E., Olmstead R.G., Morgan D., Les D.H., Mishler B.D., Duvall M.R., Price R.A., Hills H.G., Qiu Y.L., Kron K.A., Rettig J.H., Kress W.J., Karol K.G., Clark W.D., Hedren M., Gaut B.S., Jasen R.K., Kim K.J., Wimpee C.F., Smith J.F., Furnier G.R., Strauss S.H., Xiang Q.Y., Plunkett G.M., Soltis P.S., Swensen S.M., Williams S.E., Gadek P.A., Quinn C.J., Eguiarte L.E., Golenberg E., Learn G.H., Graham S.W., Barrett S.C.H., Dayanandan S., Albert V.A. 1993. Phylogenetics of seed plants: An analisis of nucleotide sequences from the plastd gene rbcL. Annals of the Missouri Botanical Garden. 80:528-580.

Crane P.R. 1985. Phylogenetic analysis of seed plants and the origin of angiosperms. Annals of the Missouri Botanical Garden 72:716-793.

Crane P.R. 1989. Paleobotanical evidence on the early radiation of nonmagnoliid angiosperms. Plant Systematics and Evolution 162:165-1991.

Crane P.R. 1995. The origin and early diversification of angiosperms. Nature 374:27-33.

Conquist A. 1981. An integrated system of classification of flowering plants. Columbia University Press. New York. Dahlgren R.M.T., Clifford G.T. and Yeo P.F. 1985. The fa- 
milies of the monocotyledons. Structure, evolution and taxonomy. Springer-Verlag. Berlin.

Doyle J.A. and Donoghue M.J. 1986. Seed plant phylogeny and the origin of angiosperms: An experimental cladistic approach. Botanical Review 52:321-431.

Doyle J.A., Donoghue M.J. and Zimmer E.A. 1994. Integration of morphological and ribosomal RNA data on the origin of angiosperms. Annats of the Missouri Botanical Garden 81:419-450.

Doyle J.A. and Donoghue M.J. 1989. Phylogenetic analysis of the angiosperms and the relationships of 'Hamamelidae'. En: Crane P.R. and Blackmore S. Eds. Evolution, systematics and fossil history of the Hamamelidae. Vol. 1. Introduction and 'lower' Hamamelidae Sistematic Association Special Vol. 40A. Clarendon Press, Oxford. pp. 17-45.

Foote M. 1991. Some notes on branching theory. Handout to participants in Paleontology Seminar (G. 536). (Inédito).

Loconte H. and Stevenson D.W. 1990. Cladistics of the spermatophyta. Brittonia 42:197-211.

Loconte H. and Stevenson D.W. 1991. Cladistics of the Magnoliidae. Cladistics 7:267-296.

Nixon K.C., Crepet W.C., Stevenson D.W. and Friis E.M. 1994. A reevaluation of seed plant phylogeny. Annals of the Missouri Botanical Garden 81:484-533.

Norell M.A. 1993. Tree-based approaches to understanding history: commments on ranks, rules, and the quality of the fossil record. American Journal of Science 293-A:407-417.

Raup D.M. 1985. Mathematical models of cladogenesis. Paleobiology 11:42-52.

Rodriguez De La Rosa R.A. and Cevallos Ferriz S.R.S. 1994. Upper Cretaceous zingiberalean fruits with in situ seeds from southeastern Coahuila, Mexico. International Journal of Plant Sciences 155:786-805.

Rothewell G.W. and Serbert R. 1994. Lignophyte phylogeny and the evolution of spermatophytes: A numerical cladistic analysis. Systematic Botany 19:443-482.

Sanderson M.J. and Donoghue M.J. 1994. Shifts in diversification rate with the origin of angiospermas. Science 264:1590-1593.

Thorne R.T. 1992. Classification and geography of the flowering plants. Botanical Review 58:225-348.

Valentine J.A. 1990. The macroevolution of clade shape. En: Ross, R.M. and Allmon W.D. Eds. Causes of evolution. A paleontological perspective. The University of Chicago Press. Chicago.

Wagner P.J. 1995. Stratigraphic tests of cladistic hypotheses. Paleobiology 21:153-178.

Yule G.U. 1924. A mathematical theory of evolution based on the conclusions of Dr. J.C. Willis F.R.S. Philosophical Transactions of the Royal Society of London. B. 213:21-87. 